\title{
Piezas de construcción y globos: propuesta didáctica para trabajar contenidos de química con futuros Maestros de Educación Primaria
}

\author{
José Manuel Montejo Bernardo
}

Universidad de Oviedo

[Recibido el 20 de abril de 2018, aceptado el 23 de agosto de 2018]

Un alto porcentaje de los alumnos del grado de Maestro en Educación Primaria proviene de la rama de letras, lo que conlleva en general un insuficiente conocimiento de ciencias y una falta de interés hacia las mismas. Ello implica una dificultad extra para entender algunos conceptos fundamentales de la química, como la estructura atómica o los enlaces químicos. En este trabajo se describe una propuesta para trabajar dichos contenidos en el aula de una forma lúdica y activa mediante el uso de piezas de construcción y globos. Las actividades propuestas despiertan el interés de los estudiantes del grado, repercuten de forma positiva en sus calificaciones y les sirven como herramienta de trabajo con sus futuros alumnos.

Palabras clave: piezas de construcción; globos; enlace químico; estructura atómica; Maestro en Primaria.

\section{Building-blocks and balloons: didactical proposal to work on chemical contents for Primary Education Teachers}

\begin{abstract}
A high percentage of students at Primary Education Teachers Degree comes from Humanities studies, which usually involves insufficient science knowledge and a lack of interest towards it. This is adding an additional difficulty for the chemical basic concepts, such as the atomic structure or the chemical bonds. In this paper, a proposal to work these contents in the classroom in a playful and active way using building-blocks and balloons is described. The proposed activities arise the students interest, have a positive impact on their academic results and serve them as a working tool with their future pupils.
\end{abstract}

Keywords: building-blocks, balloons, chemical bond, atomic structure; Primary Education Teacher

Para citar el artículo. Montejo Bernardo, J.M (2018). Piezas de construcción y globos: propuesta didáctica para trabajar contenidos de química con futuros Maestros de Educación Primaria. Ápice. Revista de Educación Científica, 2(2), 69-79. DOI: https://doi.org/10.17979/arec.2018.2.2.3438

Contacto.montejojose@uniovi.es 


\section{Introducción}

Habitualmente el átomo se representa según el modelo planetario o de Rutherford (Figura 1), con una parte central formada por protones y neutrones y con los electrones girando alrededor en órbitas elípticas, si bien el propio Rutherford no dice nada en sus trabajos sobre la disposición de los electrones en órbitas (Doménech-Blanco, SavallAlemany, y Martínez Torregrosa, 2013), y además el neutrón no fue descubierto hasta varios años después. Este es además el modelo que aparece de forma habitual en los materiales educativos sobre el átomo orientados a niños de corta edad, por ejemplo en la web de la Consejería de Educación y Universidades de la comunidad canaria (http:// www3.gobiernodecanarias.org/medusa/ecoescuela/recursoseducativos/2011/01/18/ los-atomos/), en el Proyecto Averroes del año 2014 de la Junta de Andalucía (ya no está disponible en la web, http://www.juntadeandalucia.es/educacion/portalaverroes), o en los trabajos de innovación llevados a cabo en algunos colegios (por ejemplo, MartínezMárquez, 2018).

Se explica así de forma sencilla la estructura del átomo: un núcleo central formado por protones $\left(\mathrm{p}^{+}\right)$y neutrones $\left(\mathrm{n}^{0}\right)$, y los electrones $\left(\mathrm{e}^{-}\right)$"dando vueltas" alrededor del núcleo en la corteza. Pero las dificultades pueden aparecen en el alumnado de magisterio a la hora de entender el porqué de la existencia de cada una de estas tres partículas subatómicas, y cuál es su papel dentro del átomo.

También puede resultar confuso el concepto del enlace químico. Al hablar de enlace se piensa en la unión de los átomos mediante "algo". Pero ese "algo" no aparece representado en el caso del enlace iónico (interacciones electroestáticas), mientras que en el caso del enlace metálico el concepto de "nube de electrones" puede ser un tanto abstracto. El enlace covalente es el más inteligible al ser representado por una rayita, pero lleva asociada la dificultad de la multiplicad (enlace doble y triple) y de las distintas geometrías que pueden adoptar las moléculas.
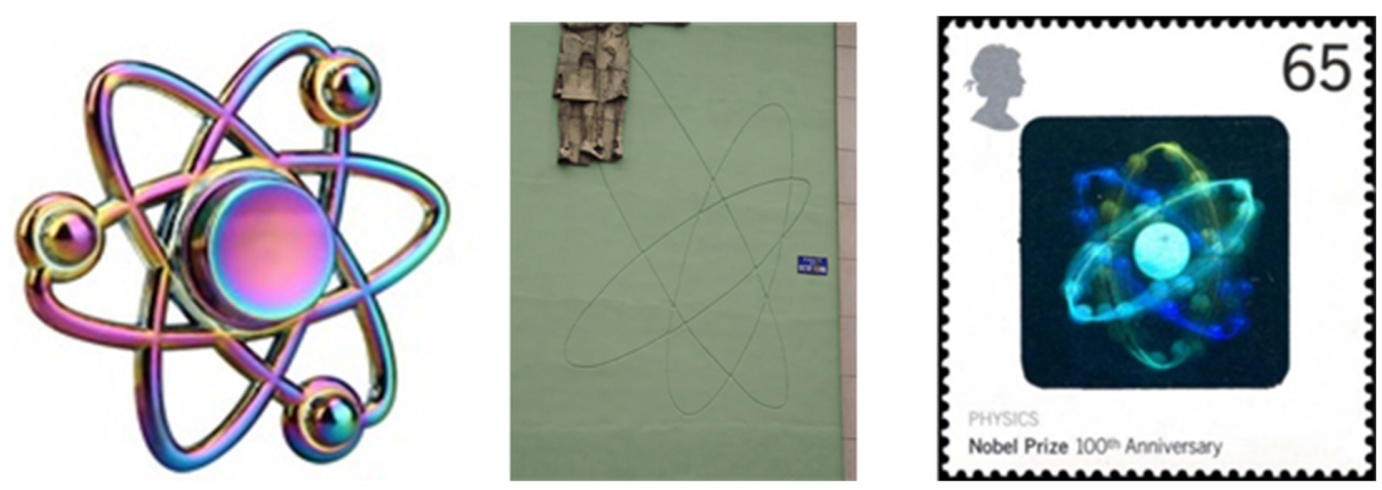

Figura 1. Ejemplos de modelo planetario del átomo en spinner, fachada de instituto y sello

En base a la experiencia del autor sobre las dificultades de los alumnos del grado de Maestro en Educación Primaria para entender aspectos relacionados con el átomo y el enlace químico, en este artículo se recoge una propuesta para trabajar en el aula la estructura interna del átomo, el papel de cada una de las partículas subatómicas y las características de los tres tipos de enlace químico, empleando para ello piezas de construcción de juguete y globos. Estos conocimientos son fundamentales para entender la constitución de la materia y para comprender otros fenómenos químicos y físicos, algunos de los cuales se abordan a lo largo del curso (reacciones químicas, corriente eléctrica, magnetismo...). 


\section{Maestros de primaria y (su falta de) conocimientos de ciencia}

La falta de una adecuada base científica en los futuros maestros de primaria es una de las principales preocupaciones referentes a su formación (Cañal de León, 2000; Del Carmen, 2010) y se viene analizando desde hace tiempo (Manzanares-Moya y GalvánBovaira, 2012; Martín del Pozo, 1998; Martínez-Chico, Jiménez-Liso y López-Gay LucioVillegas, 2014; Paixao y Cachapuz, 1999). La formación que reciben en ciencias es escasa y repercute en una deficiente preparación y en una falta de sensibilización hacia esta materia (Oliva-Martínez y Acevedo-Díaz, 2005). El problema se agrava por la baja predisposición de los estudiantes hacia las ciencias, alegando la mayoría que "son de letras" (Vílchez-González, Carrillo-Rosúa, Rodríguez-Sabiote y Jiménez-Tejada, 2015) con lo que justifican su escaso dominio de la materia o el hecho de que "no se les den bien" los contenidos de ciencias.

Considerando estas circunstancias varios autores han planteado propuestas para trabajar contenidos específicos con los estudiantes de magisterio, como el cambio químico (Martín del Pozo, 1998), las sustancias puras y mezclas (Benarroch, 2010), la energía (Ibáñez Plana y Barrau, 2014) o la estructura de átomos y moléculas (Moreno Gómez, 2015). El presente trabajo está enfocado en esas mismas líneas de actuación, para el estudio de la estructura del átomo y del enlace químico. También se han propuesto actividades alternativas, como los rincones de trabajo (Vicente-Rodado, López-Luengo, y Vallés-Rapp, 2014) o el trasladar en ocasiones la clase a un museo de ciencias (Morentin-Pascual, y Guisasola-Aranzabal, 2014).

\section{Estudiar el átomo y el enlace químico con piezas de construcción y globos.}

La idea es utilizar las piezas de construcción como modelo de protones y neutrones para explicar la estructura del átomo, y emplear los globos como si fueran electrones para mostrar las características de los tres tipos de enlace químico. El manejo de analogías es una práctica habitual en la enseñanza de las ciencias (Aragón, Bonat, Oliva y Mateo, 1999; González-González, Fernández-González y Moreno-Jiménez, 2003) ya que pueden ayudar al acercamiento de conceptos complejos a los alumnos, y las analogías de tipo estructural como las que aquí se utilizan, con objetos cotidianos, son especialmente recomendadas para la etapa de primaria (Oliva, Aragón, y Jiménez-Tenorio, 2015). El propio concepto de enlace químico es habitualmente descrito en los libros como una cuerda elástica, un muelle, un hueso compartido por dos perros, una red de pesca, etc. (De Asís-Iglesias, 2010). Sin embargo, lo que aquí se pretende, es que las analogías no sirvan solo para describir o representar el átomo y los enlaces, sino también para entender cómo se constituyen y las implicaciones químicas y físicas que ello conlleva. Se pretende que sean analogías descriptivas pero también explicativas.

Las piezas de construcción (LEGO ${ }^{\circledR}$, TENTE $^{\circledR}$, etc...) son un recurso común en la clase de química para explicar la formación de moléculas (Mans i Teixidó, 2014), las reacciones químicas y su estequiometria (Witzel, 2002) o las formas alotrópicas de los elementos (Childs, 2009). Su empleo se justifica por ser un material cotidiano, por el atractivo añadido de estar asociado con el juego y por ser más barato (y en ocasiones más versátil) que los kits comerciales de modelos moleculares. Los globos por su parte se usan también habitualmente para mostrar diversas propiedades y leyes de los gases o para ilustrar el resultado de ciertas reacciones químicas. Un listado detallado de experiencias se puede encontrar en el trabajo de profesor Williams (2005). También permiten realizar experimentos sencillos con los que mostrar fenómenos físicos como la conductividad térmica, la absorción de la luz o el efecto Joule (García-Molina, 2007). 
Con esta propuesta se pretende incidir especialmente en la parte final de la frase de Confucio "Me lo contaron y lo olvidé, lo vi y lo entendí, lo hice y lo aprendí". "Me lo contaron y lo olvidé" se correspondería con la habitual exposición en el aula por parte del profesor; "lo vi y lo entendí" sería el uso de material gráfico (esquemas, figuras, animaciones, modelos moleculares de plástico, etc.), y "lo hice y lo aprendí" es la participación activa de los alumnos construyendo los núcleos atómicos con las piezas de construcción, y adoptando el rol de átomos de diferentes elementos para formar enlaces mediante sus electrones (globos).

Asimismo, este trabajo está pensado y diseñado para que pueda ser posteriormente llevada a la práctica por los estudiantes del grado con sus futuros alumnos de primaria. Por una parte por el hecho de utilizar materiales asequibles, baratos, carentes de peligrosidad y que además presentan un componente lúdico, y por otra parte porque el grado de profundidad con el que se trate cada uno de los conceptos se puede adecuar a las características de la clase. Así por ejemplo para el caso de la estructura del átomo se puede trabajar desde el nivel más sencillo (todos los átomos están formados por el mismo tipo de "ladrillos") hasta el más complejo (presencia de neutrones y existencia de isótopos). En lo referente al enlace, la explicación puede limitarse a que todos los materiales son el resultado de la unión de sus átomos mediante un mismo tipo de partícula (el electrón), o se puede llegar al punto de trabajar incluso geometrías moleculares o asimilar un aula a la estructura ordenada de un cristal. En todos los casos, los alumnos van adquiriendo un cierto vocabulario técnico y empiezan a vislumbrar la existencia de una realidad que está más allá de lo que se ve a simple vista.

\section{Justificación y desarrollo de la propuesta en clase}

Esta actividad se viene realizando desde el curso 2015/2016 en "Didáctica de las Ciencias Experimentales" (anual, 6 créditos), que se imparte en 3ㅇ del Grado de Maestro en Educación Primaria, y que es la única asignatura del grado en la que se incluyen contenidos de física y química. El temario que se trabaja está diseñado acorde con los bloques 4 (Materia y Energía) y 5 (La tecnología, objetos y máquinas) del currículo básico de la Educación Primaria (BOE, 2014). En concreto, con la presente propuesta se pretende ahondar con los estudiantes del Grado en uno de los conceptos fundamentales que posteriormente ellos habrán de trabajar con sus futuros alumnos, tal como se señala en el Anexo I del mencionado currículo: la materia.

Las actividades aquí detalladas se incluyen dentro del primer tema de la asignatura, "Átomos, moléculas y cristales", en el que se explican conceptos fundamentales que van a ayudar a los estudiantes de magisterio a entender diversos conceptos de temas posteriores, tanto de química (la materia, reacciones químicas, mezclas...) como de física (cambios de fase, corriente eléctrica, magnetismo, conducción del calor...).

Por otra parte, desde hace ya varios años, el CSIC lleva implementando cursos de formación sobre ciencia para maestros de Primaria mediante su programa "EI CSIC en la Escuela" (http://www.csicenlaescuela.csic.es/escuela.htm) y en palabras de unos de los colaboradores del programa: "hemos llegado a la conclusión de la necesidad de empezar siempre nuestra labor formativa con aplicaciones al aula de la teoría atómico-molecular." (Moreno Gómez, 2015). La idea es pues, que los futuros estudiantes del Grado de Maestro en Educación Primaria tengan suficiente dominio del tema como para poder desarrollarlo de forma autónoma en el aula.

La propuesta se lleva a cabo con tres grupos por curso, con aproximadamente $45-50$ alumnos por grupo y en dos de las sesiones de teoría, de una hora de duración cada una. La 
mecánica de cada una de las dinámicas es muy similar. Se comienza con una breve introducción teórica para presentar los conceptos a tratar y a continuación se pasa a la parte manipulativa, en la que se van representando cada uno de ellos, el profesor va planteando preguntas y las respuestas van surgiendo en base a conocimientos previos de los alumnos y a lo que se está explicando en ese momento.

\section{El átomo: las partículas subatómicas}

Primero se describen brevemente las dos partes del átomo, núcleo y corteza y seguidamente se pasa a trabajar su estructura y el papel de cada partícula subatómica. Se utilizan piezas rojas o moradas para los protones y blancas para los neutrones, ambas de igual tamaño.

La primera idea planteada es que "lo que determina de qué elemento es un átomo es su número de protones". Se van "construyendo" diferentes "átomos": con una pieza roja (un protón) un átomo de hidrógeno $(\mathrm{H})$, con dos piezas uno de helio $(\mathrm{He})$, con seis uno de carbono (C) etc. Se pueden construir apilando las piezas una encima de otra o formando estructuras diferentes para cada elemento para dejar más clara la diferencia entre ellos (Figuras 2a, b).

Surge entonces la cuestión de la estabilidad de esos átomos. Cuerpos con carga de igual signo se repelen y átomos de sólo protones serían inestables y se desintegrarían. La respuesta a ese "misterio" justifica y explica la presencia de los neutrones en el núcleo atómico: "Ios neutrones aportan estabilidad al átomo minimizando la repulsión entre los protones" ${ }^{1}$. Para ilustrarlo, se intercalan neutrones entre los protones de los "átomos" creados anteriormente (Figura 2c).
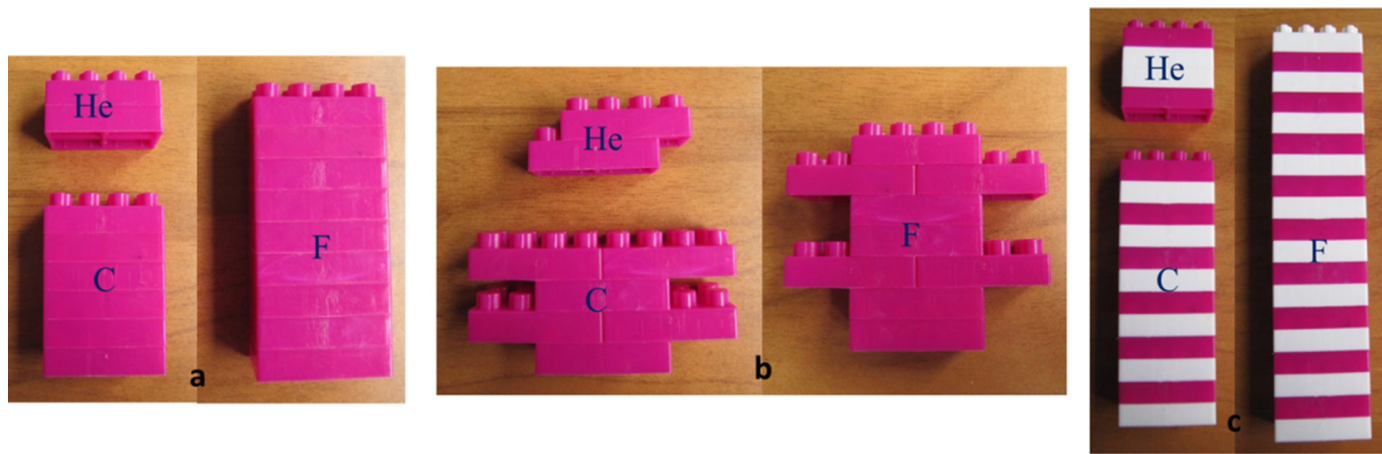

Figura 2. Construcción de átomos de He, C y F con bloques de construcción (detalles en el texto)

Puesto que su número no influye en el tipo de elemento, átomos de un mismo elemento pueden tener diferente número de neutrones. Se introduce así el concepto de isótopos y se ejemplifica con los elementos hidrógeno y carbono (Figura 3), por ser los que con mayor frecuencia aparecen en el lenguaje "cotidiano" (el deuterio y el carbono-14).

Una nueva cuestión, "¿cómo pueden unirse los átomos para formar todo lo que nos rodea si presentan cargas del mismo signo, y por tanto tenderían a alejarse unos de otros?". La respuesta sirve para introducir el electrón y de paso explicar una de sus funciones: "el átomo es neutro, tiene tantos protones (positivos) como electrones (negativos)". Se indica además que la existencia de los iones (átomos que han ganado o perdido electro-

1 Una explicación más detallada requiere el concepto de fuerza nuclear fuerte, fuera de los límites del curso. 
nes), pone de manifiesto que la naturaleza de un átomo (es decir, de qué elemento es) no depende del número de electrones que tenga.

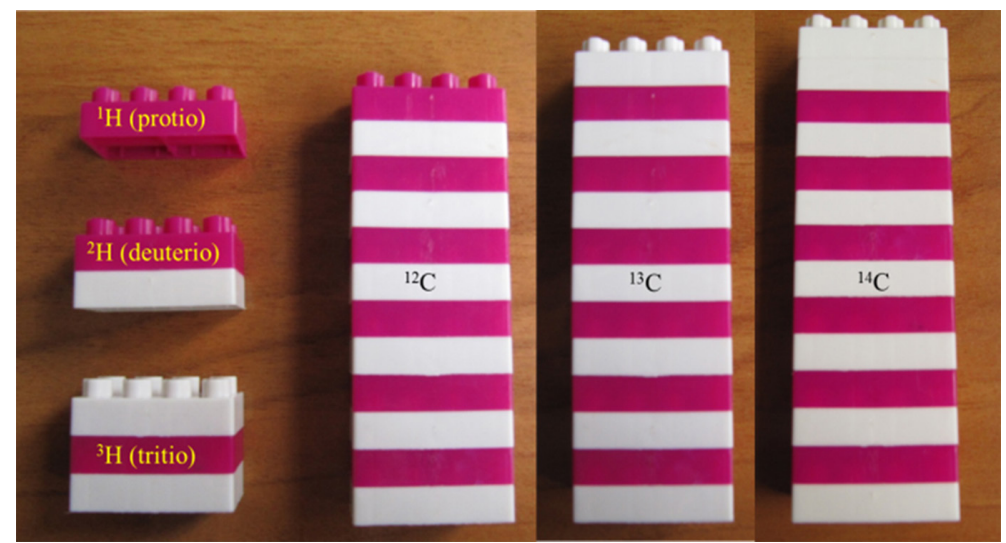

Figura 3. Isótopos del Hidrógeno y el Carbono con bloques de construcción

Se indica además que en realidad los electrones no se encuentran en órbitas (líneas) sino en regiones (volumen) alrededor del núcleo atómico denominadas orbitales y que pueden adoptar diversas formas. Para mostrar de un modo más realista "la forma" de los átomos (Castelvecchi, 2010) se introduce la segunda herramienta de esta propuesta: los globos. Con ellos se puede representar de una forma sencilla los orbitales $s, p$ y $d$ como ejemplo (Figura 4).

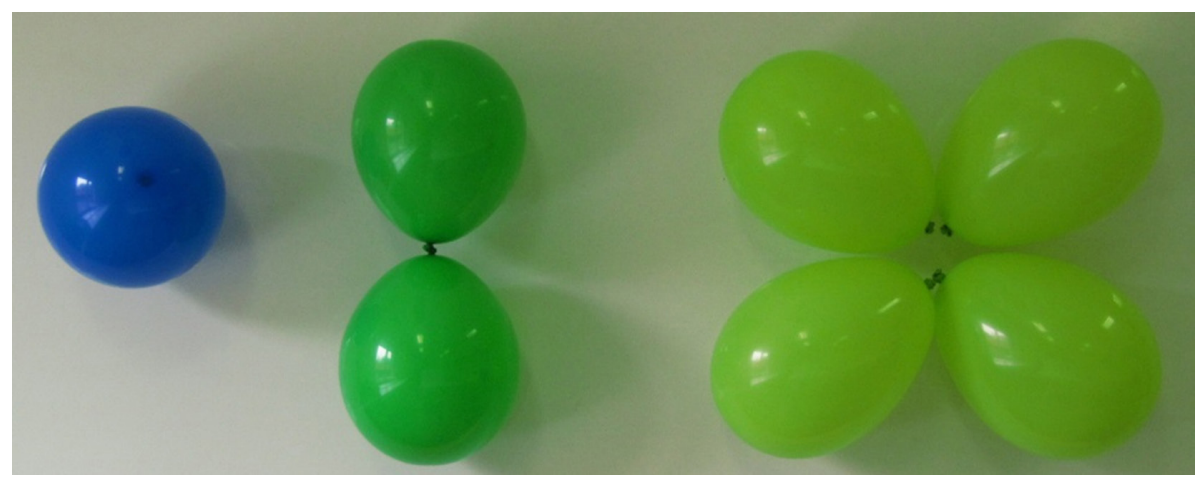

Figura 4. Representación con globos de orbitales $s, p_{y} y d_{x z}$.

\section{El enlace químico: el electrón como protagonista}

Para estudiar el papel de los electrones en la formación de los enlaces químicos los alumnos asumen el rol de átomos de diferentes elementos, y se utilizan los globos a modo de electrones. Antes de escenificar cada tipo de enlace se comentan algunas ideas previas:

1) El tipo de enlace depende de los elementos involucrados, en esencia, de su naturaleza como metales o no metales.

2) De todos los electrones que tiene cada átomo, solo algunos participan en la formación de enlaces, los denominados electrones de valencia.

3) Se hará un enfoque cualitativo del enlace, sin entrar en detalles de tipo cuantitativo.

Se empieza con el enlace iónico. Primero una breve explicación de sus características generales, metal con no metal, atracción electrostática entre anión y catión y formación de cristales, y a continuación se representa la formación de la sal de cocina (halita, $\mathrm{NaCl}$ ). 
Varios alumnos hacen de átomos de sodio, con un electrón de valencia, mientras que un número igual de alumnos hacen de átomos de cloro. Puestos frente a frente en una zona del aula, cada átomo de cloro le quita su electrón a un átomo de sodio (formación de los iones), estos intentan recuperarlo forcejeando con los átomos de sodio, permaneciendo unidos en ese forcejeo (atracción electrostática). De este modo se tiene una distribución de iones y cationes interaccionando entre ellos, que se puede asimilar a la formación final del cristal iónico.

Se sigue con el enlace metálico. Igual que antes, una breve explicación de sus características generales: solo se da en metales, los átomos pierden uno o más electrones, nube de electrones entre los cationes y formación de cristales. Ahora cada uno de los alumnos sentados en las bancadas del aula es un átomo de plata. Están dispuestos de forma ordenada de modo que representan "un cristal metálico" (si les resulta más sencillo pueden verlo como un lingote de plata). A cada uno (o a unos pocos, el efecto es el mismo y sirve para la explicación) se le entrega un globo que será el electrón que aportarán para formar el enlace metálico. A una señal del profesor los lanzan al aire (los alumnos se convierten en ese momento en cationes $\mathrm{Ag}^{+}$) y los golpean para que se muevan entre todos ellos (Figura 5). Esa nube de electrones en continuo movimiento entre los cationes de plata es el enlace metálico y mantiene unidos a los átomos del metal.

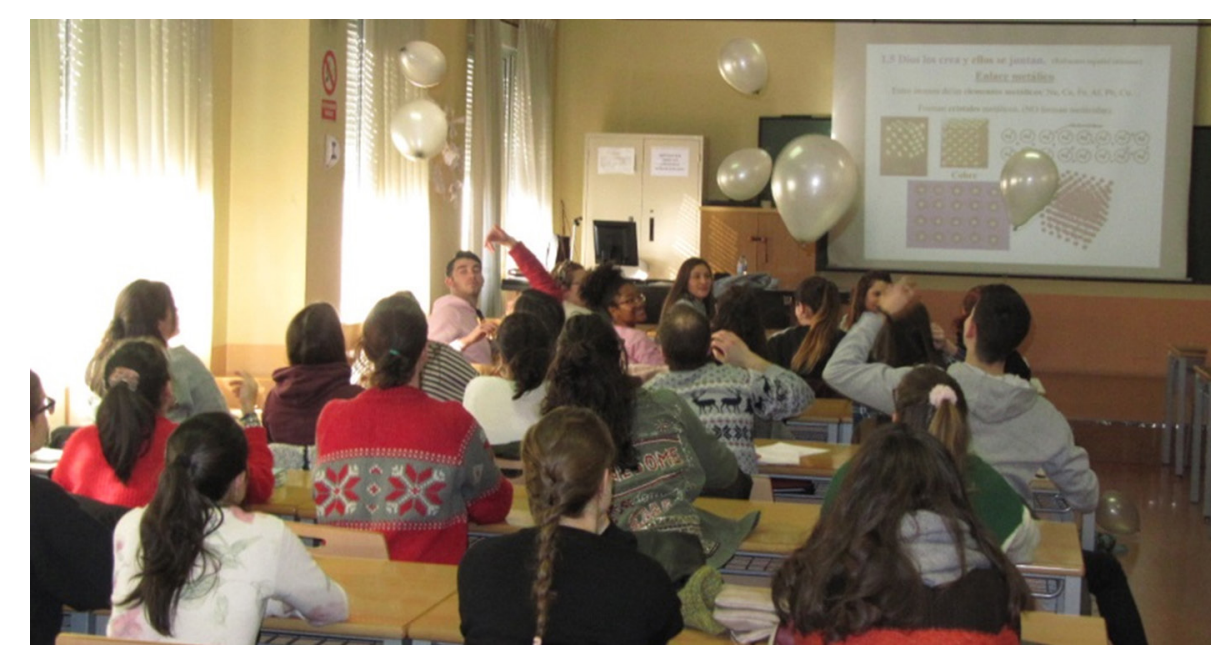

Figura 5. Representación en el aula del enlace metálico

Y se termina con el enlace covalente. Primero la breve explicación de sus características fundamentales: entre no metales, cada rayita representa dos electrones compartidos por los dos átomos unidos, y (casi siempre) conduce a la formación de entidades discretas (moléculas) en lugar de formar cristales. ${ }^{2}$

Con solo dos alumnos-átomo de hidrógeno (cada uno de ellos con un electrón para formar enlace) y dos alumnos-átomo de oxigeno (cada uno de ellos con dos electrones para compartir) se puede estudiar el orden de enlace y la geometría molecular a partir de moléculas sencillas. Para ejemplificar el orden de enlace y la geometría más simple se empieza por la molécula más pequeña posible, el $\mathrm{H}_{2}$, de geometría lineal y enlace sencillo, y a continuación se representa el $\mathrm{O}_{2}$, de igual geometría pero con enlace doble (Figura 6). Podría ilustrarse también el triple enlace con la molécula de $\mathrm{N}_{2}$, pero ello implica sujetar un tercer

2 Se indica que hay excepciones en las que se forman directamente cristales covalentes. Se muestran los ejemplos más comunes para ellos: el diamante, el grafito, el grafeno y el cuarzo. 
electrón con la boca, con las rodillas o atado al calzado. Para trabajar sobre la geometría molecular se representa la molécula de agua (Figura 7) y se plantea a los alumnos cómo sería la estructura del agua oxigenada $\mathrm{H}_{2} \mathrm{O}_{2}$, sabiendo que el hidrógeno forma siempre un único enlace y que el oxígeno se enlaza a dos átomos de forma angular. De las dos posibles conformaciones (denominadas "de silla" o "de bote") se indica que por razones estéricas ( $\sin$ entrar en detalles) la correcta es la primera.

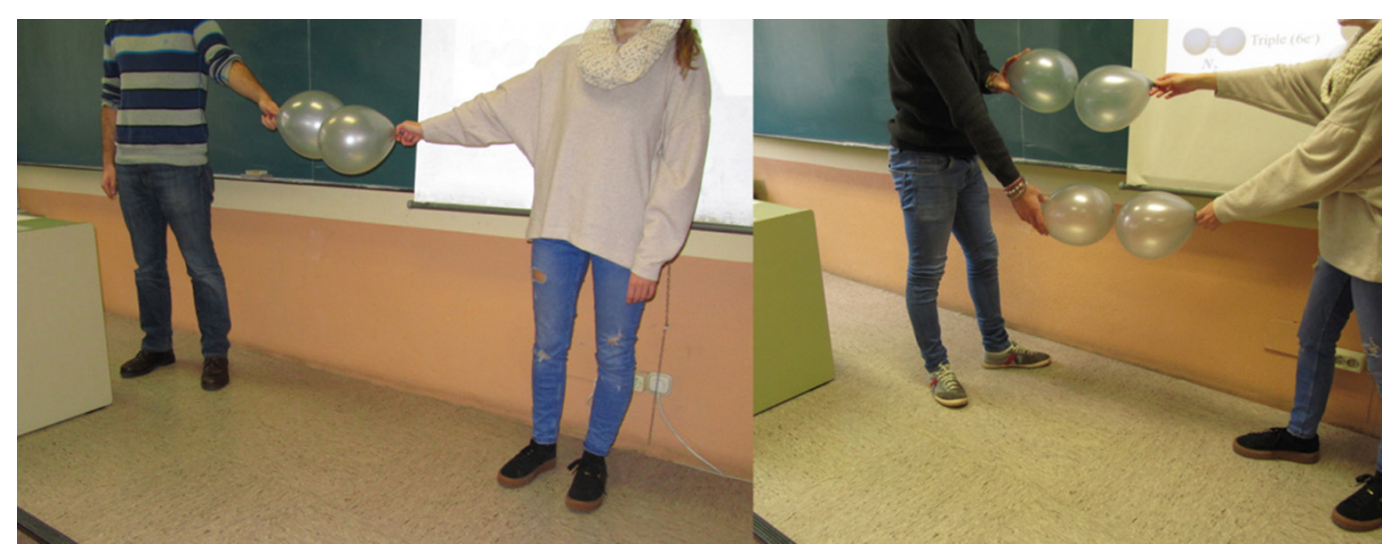

Figura 6. Representación en el aula de enlace sencillo $\left(\mathrm{H}_{2}\right)$ y enlace doble $\left(\mathrm{O}_{2}\right)$

Este trabajo con moléculas se puede complementar con el uso de un kit de modelos moleculares, aprovechando para mostrar la geometría de otras moléculas cotidianas como el metano $\left(\mathrm{CH}_{4}\right)$, el etanol $\left(\mathrm{CH}_{3} \mathrm{CH}_{2} \mathrm{OH}\right)$, el dióxido de carbono $\left(\mathrm{CO}_{2}\right)$ o el ozono $\left(\mathrm{O}_{3}\right)$, recordando que cada enlace representa siempre dos electrones compartidos.

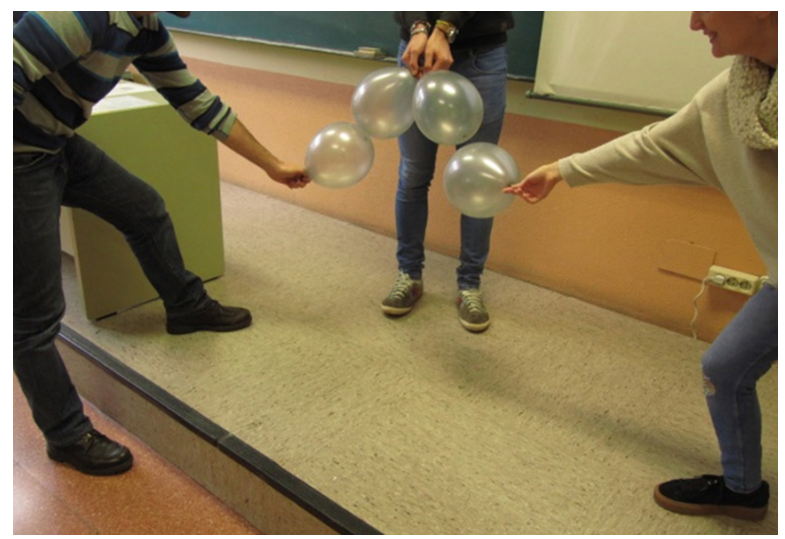

Figura 7. Representación en el aula de la molécula de $\mathrm{H}_{2} \mathrm{O}$

\section{Análisis de la propuesta y conclusión}

La presente propuesta tiene una serie de aspectos positivos que se han ido observando año tras año. Entrar en el aula con piezas de construcción y globos despierta la curiosidad de los alumnos y capta de inmediato su atención. Prueba de ello es la inmediata solicitud de varios alumnos en querer participar, incluso antes de saber sobre qué vamos a trabajar. Por otra parte, de la primera a la segunda sesión aumenta el número de alumnos en clase y este efecto se mantiene incluso varias sesiones más a la espera de nuevas actividades. Esta tendencia se mantuvo en los dos cursos en los que se llevó a cabo la propuesta y se dio en los tres grupos de clase. 
Con esta dinámica, durante el resto del curso no se habla de "lo dicho" en clase sino de "lo hecho" en clase. Las piezas de construcción y los globos permiten "poner cara" a los conceptos de átomo, molécula, part culas subatómicas y cristales, lo que favorece que los alumnos recuerden a qué se refiere el profesor cuando los nombra, y facilita la comprensión de nuevos conceptos (corriente eléctrica, reacciones nucleares, etc.), contando siempre con la posibilidad de reutilizar las piezas y los globos para explicar de forma gráfica y manipulativa esos nuevos conceptos.

En el aspecto meramente formal, la presencia de protones, neutrones y electrones en el átomo se ve ahora como algo necesario ya que cada uno de ellos juega un papel determinado. Estos conocimientos sirven para poder entender otros conceptos que van apareciendo posteriormente, tales como la formación de iones, la existencia de isótopos, o las reacciones nucleares. Asimismo permiten explicar en clase fenómenos como la obtención de energía en el sol mediante fusión nuclear (dos átomos de $\mathrm{H}$ para dar un átomo de $\mathrm{He}^{3}$ ) o la datación de materia orgánica por la técnica del ${ }^{14} \mathrm{C}$ (basada en la transmutación de ese isótopo en un átomo de nitrógeno por la "transformación" de uno de sus neutrones en un protón ${ }^{4}$.

Por su parte, la naturaleza de cada tipo de enlace queda explicada por la distinta forma en la que están repartidos los electrones entre los átomos en cada caso. En base a esto se puede explicar a lo largo del curso fenómenos como las reacciones químicas, la geometría de moléculas sencillas, la conductividad eléctrica de los metales, o la imposibilidad de conseguir la transmutación de elementos mediante reacciones químicas (piedra filosofal). También permite explicar cuestiones más mundanas, como que una pulsera de oro o un anillo de plata son cristales, por qué un lápiz escribe sobre una hoja en blanco, o cómo actúa la lejía para blanquear la ropa.

Otro argumento positivo de la propuesta son los resultados en los exámenes. Al principio de curso se realiza un cuestionario anónimo de tipo test sobre conocimientos de física y química. Una de las cuestiones planteadas es "¿Qué partícula forma los enlaces entre los átomos?". El porcentaje de respuestas correctas fue de un $21.8 \%$ para el curso 2015/2016 (sobre 147 alumnos presentes ese día en clase) y de un 28.8\% para el curso 2016/2017 (sobre 111 alumnos presentes). Esa misma cuestión se incluyó en los exámenes de mayo2016 y mayo-2017, y los porcentajes de respuestas correctas se triplicaron, llegando a un $78.7 \%$ en el primero (sobre 155 alumnos presentados) y un $79.4 \%$ en el segundo (sobre 141 alumnos presentados).

Por otra parte, en la convocatorias de junio se planteó la cuestión "¿Qué partícula subatómica participa en las reacciones químicas?" y el porcentaje de acierto alcanzó un $76.7 \%$ (sobre 90 alumnos presentados) y un 69.6\% (sobre 79 alumnos) respectivamente.

En definitiva, las actividades aquí presentadas permiten introducir en el aula de una forma ordenada y lúdica conceptos un tanto abstractos y confusos en ocasiones para el alumnado. Su implicación directa en la "construcción" de los mismos permite un mejor entendimiento por su parte y allana el camino para la explicación posterior de conceptos y fenómenos químicos más elaborados.

3 En realidad estaríamos contando solo el principio y el final. El proceso completo es algo más complejo e implica la presencia de más de un isotopo del hidrogeno y del helio, pero es un buen ejemplo para ver la relación entre número de protones y elementos diferentes.

4 Proceso denominado desintegración beta, que incluye también la emisión de un electrón y un antineutrino electrónico. 


\section{Referencias bibliográficas}

Aragón, M. M.; Bonat, M.; Oliva, J. M. y Mateo, J. (1999). Las analogías como recurso didáctico en la enseñanza de las ciencias. Alambique, 22, 109-115.

Benarroch, A. (2010). El aire y el agua: ¿sustancias puras o mezclas? Una sesión de clase para futuros maestros fundamentada en la investigación didáctica. Alambique, 63, 91-105.

BOE. (2014). Real Decreto 126/2014, de 28 de febrero, por el que se establece el currículo básico de la Educación Primaria. BOE, 52 (1 marzo), 19349-19420.

Cañal de León, P. (2000). El conocimiento profesional sobre las ciencias y la alfabetización científica en primaria. Alambique, 24, 46-56.

Castelvecchi, D. (2010). La forma de los átomos. Investigación y Ciencia, 410(Febrero), 8-9.

Childs, P. E. (2009). Teaching about elements compounds and mixtures. Chemistry in Action, 89, 19-25.

De Asís-lglesias, F. (2010). Analogías utilizadas habitualmente en la enseñanza de química básica en la ESO. Alambique, 64, 86-98.

Del Carmen, L. M. (2010). Formar maestros competentes: un reto difícil para el sistema educativo. Aula de Innovación Educativa, 195, 47-52.

Doménech-Blanco, J. L.; Savall-Alemany, F. y Martínez Torregrosa, J. (2013). ¿Los libros de texto de bachillerato introducen adecuadamente los modelos atómicos de Thomson y Rutherford? Enseñanza de las Ciencias, 31(1), 29-43. DOI: https://doi. org $/ 10.5565 / \mathrm{rev} / \mathrm{ec} / \mathrm{v} 31 \mathrm{n} 1.764$

García-Molina, R. (2007). Cuatro experiencias sorprendentes y sencillas con globos. Revista EUREKA de Enseñanza y Divulgación de las Ciencias, 4(2), 343-345. DOI: http://dx.doi.org/10.25267/Rev_Eureka_ensen_divulg_cienc.2007.v4.i2.11

González-González, B. M.; Fernández-González, J. y Moreno-Jiménez, T. (2003). Las analogías como modelo y como recurso en la enseñanza de las ciencias. Alambique, $35,82-89$.

Ibáñez Plana, M y Barrau, J. (2014). El balance energético en escenarios reales. Propuesta didáctica en la formación inicial de maestros. Revista EUREKA de Enseñanza y Divulgación de las Ciencias, 11(2), 216-230. DOI:http://dx.doi.org/10.25267/ Rev_Eureka_ensen_divulg_cienc.2014.v11.i2.07

Mans i Teixidó, C. (2014). De cómo el átomo se convierte en una pieza de LEGO. Una visión crítica. EduQ, 19, 33-38.

Manzanares-Moya, A. y Galván-Bovaira, M. J. (2012). La formación permanente del profesorado de educación infantil y primaria a través de los centros de profesores. Un modelo de evaluación. Revista de Educación, 359, 431-455. DOI: https://doi. org/10.4438/1988-592X-RE-2011-359-101

Martín del Pozo, R. (1998). La formación inicial de maestros sobre los contenidos escolares. El caso del cambio químico. Investigación en la escuela, 35, 21-32.

Martínez-Chico, M.; Jiménez-Liso, M. R. y López-Gay Lucio-Villegas, R. (2014). La indagación en las propuestas de formación inicial de maestros: análisis de entrevistas a los formadores de Didáctica de las Ciencias Experimentales. Enseñanza de las Ciencias, 32(3), 591-608. DOI: https://doi.org/10.5565/rev/ensciencias.1376 
Martínez-Márquez, M. L. (2018). Trabajo cooperativo para estudiar las teorías atómicas y reacciones químicas en Primaria. Recuperado de https://cesdonbosco.com/grados/ vida-universitaria/602-cooperacion-quimica.html

Moreno Gómez, E. (2015). El modelo atómico de la materia en la formación científica del profesorado de las primeras etapas educativas. Anales de Química, 111(3), 181-187.

Morentin-Pascual, M. y Guisasola-Aranzabal, J. (2014). La visita a un museo de ciencias en la formación inicial del profesorado de Educación Primaria. Revista EUREKA de Enseñanza y Divulgación de las Ciencias, 11(3), 364-380. DOI: http://dx.doi. org/10.25267/Rev_Eureka_ensen_divulg_cienc.2014.v11.i3.07

Oliva, J. M.; Aragón, L.y Jiménez-Tenorio, N. (2015). Analogías y progresión del conocimiento del alumnado en la clase de ciencias. Alambique, 79, 35-44.

Oliva-Martínez, J.M. y Acevedo-Díaz, J. A. (2005). La enseñanza de las ciencias en primaria y secundaria hoy. Algunas propuestas de futuro. Revista EUREKA de Enseñanza y Divulgación de las Ciencias, 2(2), 241-250. DOI: http://dx.doi.org/10.25267/ Rev_Eureka_ensen_divulg_cienc.2005.v2.i2.10

Paixao, M. F. y Cachapuz, A. (1999). La enseñanza de las ciencias y la formación de profesores de enseñanza primaria para la reforma curricular: de la teoría a la práctica. Enseñanza de las Ciencias, 17(1), 69-77. Recuperado de: http://www.raco. cat/index.php/Ensenanza/article/view/21561

Vicente-Rodado, F.; López-Luengo, M.A. y Vallés-Rapp, C. (2014). Los rincones de trabajo como estrategia en la formación de maestros para la enseñanza de ciencias y su didáctica. Tendencias Pedagógicas, 23, 109-126.

Vílchez-González, J. M.; Carrillo-Rosúa, J.; Rodríguez-Sabiote, C. y Jiménez-Tejada, M. P. (2015). Imagen de ciencia de estudiantes de Magisterio. Didáctica de las Ciencias Experimentales y Sociales, 29, 157-172. DOI: https://doi.org/10.7203/dces.29.4283

Williams, K. R. (2005). Balloon. Toy of many colors. Journal of Chemical Education, 82(10), 1448-1449. DOI: https://doi.org/10.1021/ed082p1448

Witzel, J. E. (2002). Lego Stoichiometry. Journal of Chemical Education, 79(3), 352A-352B. DOI: https://doi.org/10.1021/ed079p352A 